\title{
Combined inhibition of PIM and CDK4/6 suppresses both mTOR signaling and Rb phosphorylation and potentiates PI3K inhibition in cancer cells
}

\author{
Lacey M. Litchfield ${ }^{1}$, Karsten Boehnke ${ }^{2}$, Manisha Brahmachary ${ }^{2}$, Cecilia Mur ${ }^{3}$, \\ Chen $\mathrm{Bi}^{1}$, Jennifer R. Stephens ${ }^{1}$, J. Michael Sauder ${ }^{4}$, Sonia M. Gutiérrez ${ }^{3}$, Ann M. \\ McNulty ${ }^{1}$, Xiang S. Ye ${ }^{5}$, Wenjuan Wu${ }^{1}$, María José Lallena ${ }^{3}$, Xueqian Gong ${ }^{1}$, Farhana \\ F. Merzoug ${ }^{1}$, Valerie M. Jansen ${ }^{1}$ and Sean G. Buchanan ${ }^{1}$ \\ ${ }^{1}$ Eli Lilly and Company, Indianapolis, IN, USA \\ ${ }^{2}$ Eli Lilly and Company, New York, NY, USA \\ ${ }^{3}$ Eli Lilly and Company, Alcobendas, Madrid, Spain \\ ${ }^{4}$ Eli Lilly and Company, San Diego, CA, USA \\ ${ }^{5}$ Eli Lilly and Company, Shanghai, China \\ Correspondence to: Sean G. Buchanan, email: buchananse@lilly.com \\ Keywords: abemaciclib; CDK4/6; PIM; mTOR; S6 \\ Received: November 04, 2019 Accepted: March 14, $2020 \quad$ Published: April 28, 2020 \\ Copyright: Litchfield et al. This is an open-access article distributed under the terms of the Creative Commons Attribution License 3.0 (CC \\ BY 3.0), which permits unrestricted use, distribution, and reproduction in any medium, provided the original author and source are credited.
}

\section{ABSTRACT}

Aberrant activation of mitogenic signaling pathways in cancer promotes growth and proliferation of cells by activating mTOR and S6 phosphorylation, and D-cyclin kinases and Rb phosphorylation, respectively. Correspondingly, inhibition of phosphorylation of both $\mathbf{R b}$ and $\mathbf{S} 6$ is required for robust anti-tumor efficacy of drugs that inhibit cell signaling. The best-established mechanism of mTOR activation in cancer is via PI3K/Akt signaling, but mTOR activity can also be stimulated by CDK4 and PIM kinases. In this study, we show that the CDK4/6 inhibitor abemaciclib inhibits PIM kinase and 56 phosphorylation in cancer cells and concurrent inhibition of PIM, CDK4, and CDK6 suppresses both S6 and Rb phosphorylation. TSC2 or PIK3CA mutations obviate the requirement for PIM kinase and circumvent the inhibition of S6 phosphorylation by abemaciclib. Combination with a PI3K inhibitor restored suppression of $\mathbf{S} 6$ phosphorylation and synergized to curtail cell growth. By combining abemaciclib with a PI3K inhibitor, three pathways (Akt, PIM, and CDK4) to mTOR activation are neutralized, suggesting a potential combination strategy for the treatment of PIK3CA-mutant ER+ breast cancer.

\section{INTRODUCTION}

Cyclin-dependent kinases (CDK) 4 and 6, which phosphorylate the retinoblastoma tumor suppressor protein $\mathrm{Rb}$ to promote $\mathrm{G} 1$ to $\mathrm{S}$ transition, are commonly dysregulated in cancer [1]. Genetic alterations that increase expression or stability of D-cyclins result in activation of D-cyclin dependent kinases, and, thus, confer dependence on CDK4/6 for continued cell cycle progression and cell proliferation [2]. D-cyclin levels are also modulated downstream of mitogen and hormone signaling pathways, including PI3K, MAPK, and ER [3-5], suggesting that aberrant activation of these pathways may also result in
CDK4/6 dependencies that could be exploited clinically. In addition to stimulating cell cycle initiation via D-cyclin dependent kinases and $\mathrm{Rb}$ phosphorylation, these signaling pathways promote cell growth via mTOR activation and S6 phosphorylation, and it has been shown that inhibition of phosphorylation of both $\mathrm{Rb}$ and $\mathrm{S} 6$ is required for robust anti-tumor efficacy of drugs that inhibit cell signaling [612]. mTOR activity can also be stimulated, independently of the PI3K pathway, by CDK4 and PIM kinases [13-16], both of which have been identified as potential mechanisms of resistance to PI3K inhibitors in breast cancer $[6,15]$.

The CDK4/6 inhibitors abemaciclib, palbociclib, and ribociclib have emerged as important new treatment 
options for $\mathrm{HR}+$, HER2- advanced breast cancer in combination with endocrine therapy and are under investigation for additional indications [17-19]. Several distinguishing characteristics of the three drugs have been reported. Abemaciclib is structurally distinct from palbociclib and ribociclib. In addition, abemaciclib has greater potency against CDK4 than CDK6 in enzymatic assays [20]. Clinically, lower rates of neutropenia result from treatment with abemaciclib allow for continuous, twice-daily dosing; palbociclib and ribociclib are administered on an intermittent dosing schedule [1, 21]. Abemaciclib has also demonstrated single-agent activity $[21,22]$ and is, uniquely among the CDK4/6 inhibitors, approved by the FDA for use as monotherapy [23], but the mechanistic basis for this activity remains to be fully understood. The approved CDK4/6 inhibitors show activity against additional kinases in in vitro assays [2427], but, for these additional targets of the drugs, direct evidence of inhibition in cells is limited, and in most cases it is unlikely that they are potently inhibited in cells at the plasma concentrations achieved at clinical doses [2, 28].

Here we show that abemaciclib can suppress the kinase activity of the oncoprotein PIM, and that, similar to PIM inhibitors, abemaciclib inhibits S6 phosphorylation in cells with wild-type PIK3CA and TSC2. Additionally, we evaluate the potential utility of concurrent treatment with abemaciclib and the PI3K inhibitor BYL719 (alpelisib) in $P I K 3 C A$ mutant breast cancer. Our results suggest that abemaciclib can inhibit the mTOR pathway independently of its effects on $\mathrm{Rb}$ and support combining abemaciclib with $\mathrm{PI} 3 \mathrm{~K} / \mathrm{mTOR}$ pathway inhibitors to fully suppress phosphorylation of S6 via multiple inputs.

\section{RESULTS}

\section{Single-agent abemaciclib rapidly inhibits mTOR signaling}

While evaluating the effects of CDK4/6 inhibitors on growth and proliferative signaling pathways, we noticed that, intriguingly, abemaciclib treatment rapidly suppressed S6 and 4EBP1 phosphorylation in several cell lines, including DMS-53 small cell lung cancer (SCLC) and MDA-MB-175 breast cancer cells (Figure 1A, Supplementary Figure 1A, 1B). In the same cell lines, palbociclib and ribociclib did not alter S6 or 4EBP1 phosphorylation, although, as with abemaciclib, $\mathrm{Rb}$ phosphorylation was inhibited. In addition to DMS53 and MDA-MB-175, inhibition of S6 phosphorylation was also observed following abemaciclib treatment in cell lines of several other cancer types, including mantle cell lymphoma (MCL; Jeko-1), pancreas cancer (MiaPaCa2), osteosarcoma (U2OS), melanoma (SK-MEL-28), nonsmall cell lung cancer (NSCLC; A549), and even Rb-null triple negative breast cancer (TNBC; MDA-MB-468) (Supplementary Figure 1C, 1D). The major metabolites of abemaciclib, M2 and M20 [28, 29], also inhibited S6 phosphorylation (Supplementary Figure 1E). In vivo, S6 phosphorylation was inhibited by abemaciclib, but not by palbociclib, in A549 xenograft tumors, while reduced $\mathrm{Rb}$ phosphorylation was evident following treatment with either drug (Figure 1B). Inhibition of S6 phosphorylation was also sustained following prolonged exposure to abemaciclib for two cell doublings, indicating that this response is durable (Supplementary Figure 1F). Longterm treatment with palbociclib did not substantially affect S6 phosphorylation, suggesting the persistent mTOR pathway suppression by abemaciclib may not have been simply an indirect consequence of cell cycle inhibition (Supplementary Figure 1F). Indeed, loss of Rb expression in DMS-53 did not alter the ability of abemaciclib to inhibit S6 phosphorylation following either 4 or $24 \mathrm{~h}$ treatment (Figure 1C). CDK4 and/or CDK6 knockdown also had only a modest effect on S6 phosphorylation, similar to the results with palbociclib and ribociclib treatment, suggesting the effects of abemaciclib on MTOR signaling may be mediated by a CDK4/6-Rb-independent mechanism (Figure 1D, Supplementary Figure 2A).

\section{PIM kinase inhibition phenocopies mTOR suppression by abemaciclib}

Another feature of abemaciclib is its reported inhibition of PIM kinases ([20], Supplementary Figure 2B). The PIM kinases, PIM1, PIM2, and PIM3, phosphorylate a diverse array of substrates regulating cell growth, and these substrates share significant overlap with known substrates of Akt signaling [16]. As potent effects of PIM inhibition on mTOR signaling (including S6 and p70S6K phosphorylation) have been reported $[30,31]$, we hypothesized that inhibition of PIM was a plausible explanation for the observed reduction in S6 phosphorylation by abemaciclib. Indeed, treatment with the PIM inhibitors PIM447 and AZD1208 closely phenocopied the ability of abemaciclib to inhibit phosphorylation of S6, p70S6K, and the PIM substrate BAD (Figure 2A). Knockdown of PIM1, 2, and 3, individually or in combination, likewise suppressed S6 and BAD phosphorylation (Figure 2B). Reduced phosphorylation of BAD was also evident following abemaciclib treatment in vivo (Supplementary Figure 2C). Inhibitors of DYRK1B (compound 33 [32]) or CDK9 (dinaciclib), additional kinases inhibited by abemaciclib in biochemical assays [20], did not impact S6 or p70S6K phosphorylation (Supplementary Figure 2D). Two additional CDK4/6 inhibitors identified during the development of abemaciclib, and closely related by chemical structure [33], likewise reduced phosphorylation of S6, p70S6K, and BAD, and were found to have activity against PIM kinases in biochemical and cellular assays (Figure 2C). Abemaciclib metabolites M2 and M20 were also found to inhibit PIM kinase (data not shown) 


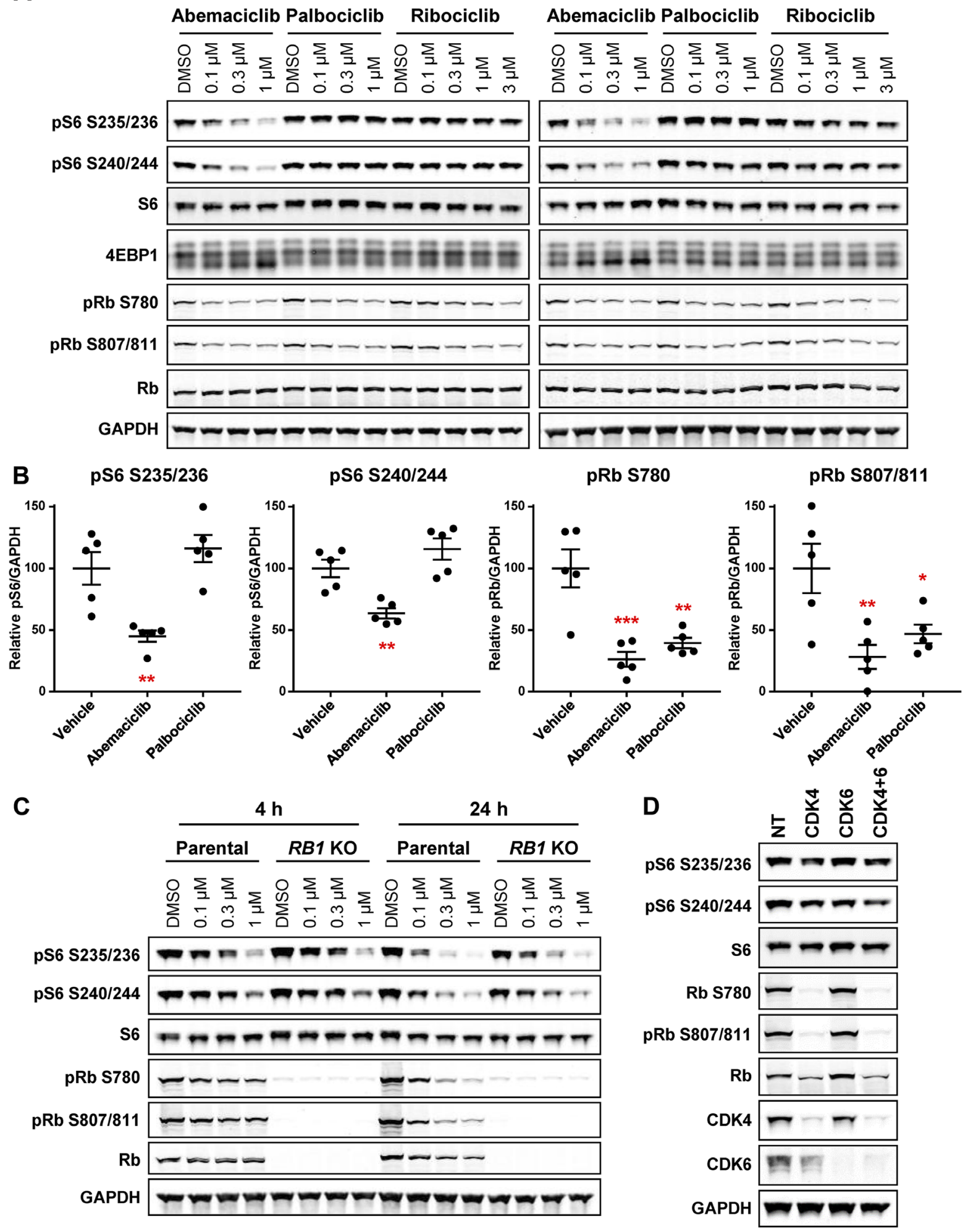

Figure 1: Abemaciclib inhibits S6 phosphorylation independent of effects on CDK4/6 and Rb. (A) DMS-53 and MDAMB-175 cells were treated with the indicated concentrations of abemaciclib, palbociclib, or ribociclib for $4 \mathrm{~h}$ and analyzed by western blot. (B) Mice bearing A549 xenograft tumors were treated with a single dose of abemaciclib or palbociclib $(50 \mathrm{mg} / \mathrm{kg}$ ). Tumors were collected $24 \mathrm{~h}$ post-treatment and analyzed by western blot. Plots indicate mean $\pm \operatorname{SEM}\left(n=5\right.$ /group), relative to vehicle control. ${ }^{*} p<0.05 ;{ }^{* *} p<$ $0.01 ; *{ }^{* * *} p<0.001$. (C) DMS-53 parental or $R B 1$ KO cells were treated with the indicated concentrations of abemaciclib for 4 or $24 \mathrm{~h}$ and analyzed by western blot. (D) DMS-53 cells were transfected with $C D K 4, C D K 6, C D K 4+C D K 6$, or non-targeting control (NT) siRNA for $48 \mathrm{~h}$ and analyzed by western blot. 
consistent with their ability to inhibit S6 phosphorylation in cells (Supplementary Figure 1E).

A structural comparison of how abemaciclib and palbociclib could bind in the ATP pocket of the PIM1 isoform allowed us to understand their different activities. We modeled abemaciclib binding to the PIM1 catalytic site. Overlaying palbociclib on this model and inspection of the catalytic Lys67 (which salt-bridges Glu89 on the C- $\alpha$-helix), gatekeeper (Leu120) and DFG domain showed multiple clashes which would prevent binding (Supplementary Figure 3). Abemaciclib, by contrast, is seen to (i) fill the ATP pocket under the p-loop, (ii) make hydrophobic contact with Val52, and (iii) make a favorable interaction with Lys67 explaining its potency against PIM kinase.

\section{Combined inhibition of CDK4/6 and PIM enhances suppression of mTOR and cell growth}

We next examined the impact of concurrent PIM and CDK4/6 inhibition by treating cells with PIM447 in combination with either abemaciclib or palbociclib. In DMS-53 cells abemaciclib monotherapy was approximately 8 -fold more potent than palbociclib monotherapy (CTG $\mathrm{IC}_{50} 68$ v $539 \mathrm{nM}$ ), despite similar inhibition of phospho-Rb (Figures 3A, 1A). The addition of PIM447 to palbociclib substantially improved potency to $100 \mathrm{nM}$, bringing it into the same range as abemaciclib monotherapy, and corresponding to significant synergy (combination index $0.115 ; 95 \%$ confidence interval 0.041 0.318) (Figure 3A). The PIM447/palbociclib combination also showed improved efficacy (\% inhibition at the plateau of the dose response curve). Addition of PIM447 to abemaciclib, on the other hand, did not improve over abemaciclib's monotherapy potency ( $\mathrm{IC}_{50} 76 \mathrm{v} 68 \mathrm{nM}$ ), and the combination was not synergistic (combination index $0.545 ; 95 \%$ confidence interval $0.124-1.786$ ). The potency improvement conferred by addition of the PIM inhibitor to palbociclib is accompanied by inhibition of S6 phosphorylation to a degree comparable to abemaciclib monotherapy (Figure 3B).

\section{PIM regulation of $m$ TOR signaling requires TSC2 and GSK3ß}

Several mechanisms have been postulated to underlie the control of mTOR signaling by PIM kinases, including phosphorylation and inactivation of mTOR suppressors PRAS40 and tuberin (TSC2) $[34,35]$. PRAS40 phosphorylation was unchanged following treatment with abemaciclib or PIM inhibitors (Supplementary Figure 4), consistent with a previous report [35]. TSC2 knockdown in DMS-53 (Figure 4A), or knockout in A549 (Figure 4B), prevented the reduction in S6 phosphorylation by abemaciclib, but not by the mTOR inhibitor everolimus (Supplementary Figure 5A,
5B), suggesting that PIM acts upstream of TSC2, although we have not been able to detect phosphorylation of TSC2 at a reported PIM-specific site (Ser1798 [35]) in DMS53 cells (data not shown). Similarly, in SNU-886, a hepatocellular carcinoma cell line with natural TSC2 loss, abemaciclib was unable to suppress S6 phosphorylation (Supplementary Figure 5C).

In addition to phosphorylation of TSC2, PIM was also reported to phosphorylate glycogen synthase kinase $3 \beta$ (GSK3 $\beta$ ) at Ser9, resulting in its inactivation [36]. As GSK3 $\beta$ has also been shown to phosphorylate and activate TSC2 to suppress mTOR signaling [37], indirect TSC2dependent mTOR regulation by PIM could also occur. Indeed, phosphorylation of GSK3 $\beta$ at Ser9 was reduced following treatment with abemaciclib (but not palbociclib; Figure 4C), indicative of GSK3 $\beta$ activation, and addition of the GSK3 inhibitor LY2090314 [38] to abemaciclib or PIM447 reversed the inhibition of S6 phosphorylation by either drug (Figure 4D). These results define GSK3 $\beta$ suppression as a target of PIM responsible for its ability to activate the mTOR pathway and GSK3 $\beta$ activation as a key mechanism of inhibition of mTOR by abemaciclib and PIM kinase inhibitors.

\section{PI3K activity compensates for PIM in PIK3CA mutant breast cancer}

Given the overlap between PIM and PI3K/Akt signaling and the identification of PIM kinases as mediators of resistance to PI3K and HER2 inhibition in breast cancer $[15,39]$, we next asked whether PI3K signaling in cell lines with PIK3CA and/or HER2 alterations would compensate for PIM. Inhibition of S6 and p70S6K phosphorylation by abemaciclib was substantially reduced in PIK3CA mut/HER2+ MDAMB-453 and PIK3CA mut T-47D, as compared to PIK3CA WT MDA-MB-175 (Figure 5A). While single-agent treatment with abemaciclib or PIM447 was unable to inhibit downstream mTOR signaling in MDA-MB-453, concurrent treatment with the PI3K inhibitor BYL719 and either drug resulted in inhibition of S6 and p70S6K phosphorylation (Figure 5B). Intriguingly, this inhibition was superior to the inhibition by BYL719 alone. Together, these data suggest that the functional redundancy of PIM and PI3K/Akt signaling could be targeted by combination therapy to counteract compensatory signaling and augment the responses achieved by single-agent treatment.

\section{Concurrent abemaciclib and BYL719 treatment synergistically inhibits cell growth}

To further explore the potential benefit of combining abemaciclib with PI3K inhibition, we tested the ability of concurrent treatment with abemaciclib and BYL719 to inhibit cell growth in panel of 31 breast cancer cell lines. The tested cell lines displayed a range of sensitivities to single- 
agent abemaciclib or BYL719 (Supplementary Figure $6 \mathrm{~A}, 6 \mathrm{~B})$. Combination treatment resulted in synergistic inhibition of cell growth in twelve cell lines (Figure 6A) and was synergistic or additive in all 9 PIK3CA mut cell lines tested. Additionally, PIM1 expression levels in PIK3CA mut cell lines correlated with the abemaciclib-BYL719 combination synergy score (Figure 6B). Confirmatory assays demonstrated synergism between abemaciclib and BYL719 in PIK3CA mut MCF-7 and T-47D cells, while the combination was additive in PIK3CA WT ZR-75-1 (Figure 6C). Similarly, the combination of a PDPK1 inhibitor (GSK2334470) and abemaciclib also synergistically inhibited growth of T-47D cells (Supplementary Figure 7A) and improved inhibition of downstream S6 and p70S6K phosphorylation (Supplementary Figure 7B), suggesting the observed effects of targeting PI3K in combination with abemaciclib may also apply to drugs targeting other nodes of the pathway [40].

\section{mTOR signaling is suppressed in breast cancer patients following abemaciclib treatment}

The inhibitory effects of abemaciclib on mTOR signaling in cancer cell lines and xenograft models all occur at drug concentrations that are readily achieved in patients receiving the recommended dose [2]. This implies

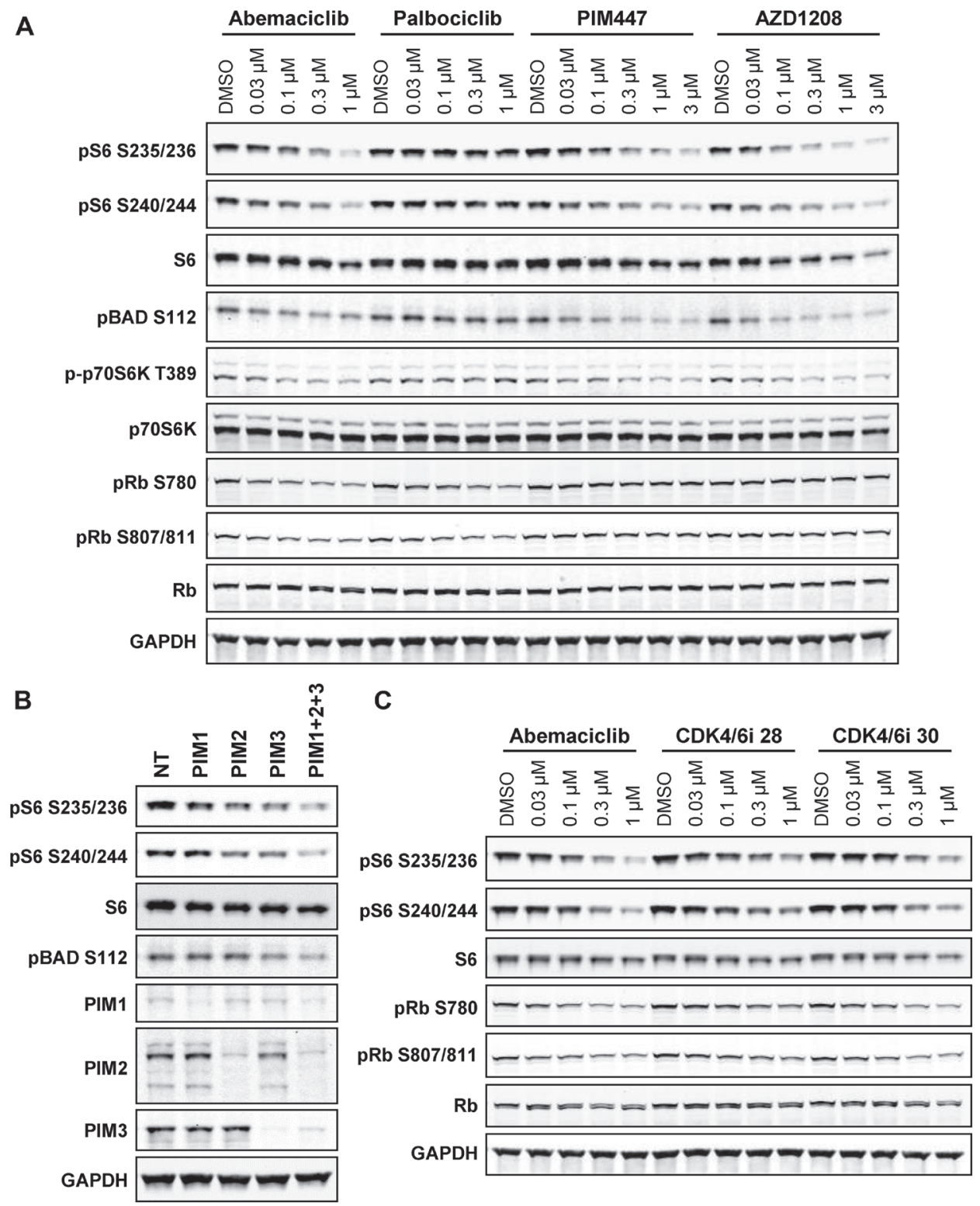

Figure 2: PIM kinase inhibition phenocopies effects of abemaciclib on mTOR signaling. (A) DMS-53 cells were treated with the indicated concentrations of abemaciclib, palbociclib, or PIM inhibitors PIM447 or AZD1208 for $4 \mathrm{~h}$ and analyzed by western blot. (B) DMS-53 cells were transfected with PIM1, PIM2, PIM3, PIM1+PIM2+PIM3, or non-targeting control (NT) siRNA for $48 \mathrm{~h}$ and analyzed by western blot. (C) DMS-53 cells were treated with the indicated concentrations of abemaciclib or two additional CDK4/6 inhibitors for $4 \mathrm{~h}$ and analyzed by western blot. 
that these effects may also be evident in patients. To test this prediction, we analyzed RNAseq data from a phase 2 study of neoadjuvant abemaciclib in postmenopausal, $\mathrm{HR}+$, HER2- breast cancer patients (neoMONARCH, NCT02441946 [41]) and identified top scoring pathways via gene set enrichment analysis (GSEA). Following two weeks of treatment with abemaciclib monotherapy, in addition to cell cycle-related signatures (including G2M checkpoint and E2F targets [41]), the mTORC1 signaling pathway was significantly downregulated (Figure 7). A similar analysis of the publicly available dataset from the NeoPalAna study (NCT01723774 [42]) did not find mTOR signaling to be significantly altered following addition of palbociclib treatment for two weeks (data not shown). These results suggest that abemaciclib's inhibition of the mTORC1 signature is not an indirect consequence of CDK4/6 inhibition and further support the conclusion that the mTOR pathway inhibitory activity of abemaciclib described in cancer cell lines also manifests in tumors from patients exposed to the drug.

\section{DISCUSSION}

Previous studies of mTOR/S6 regulation by CDK4/6, $\mathrm{Rb}$, and the cell cycle have yielded somewhat conflicting reports across various cellular contexts and require
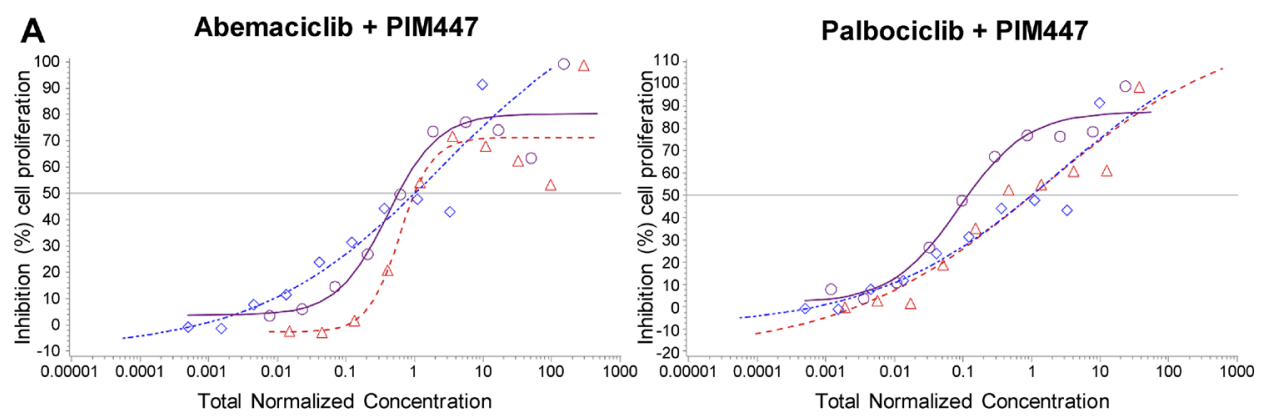

\begin{tabular}{lc}
\hline Compound & ${\text { Absolute } \mathrm{IC}_{50}(\mu \mathrm{M})}$ \\
\hline$\triangle$ Abemaciclib & 0.068 \\
$\diamond$ PIM447 & 2.027 \\
O Combination & 0.076 \\
Abemaciclib+PIM447 & \\
\hline $\mathrm{CI}$ & 0.545
\end{tabular}

\begin{tabular}{lc}
\hline \multicolumn{1}{c}{ Compound } & ${\text { Absolute } \mathrm{IC}_{50}(\mu \mathrm{M})}^{\Delta \text { Palbociclib }}$ \\
$\diamond$ PIM447 & 0.539 \\
O Combination & 2.027 \\
Palbociclib+PIM447 & 0.100 \\
\hline $\mathrm{Cl}$ & 0.115
\end{tabular}

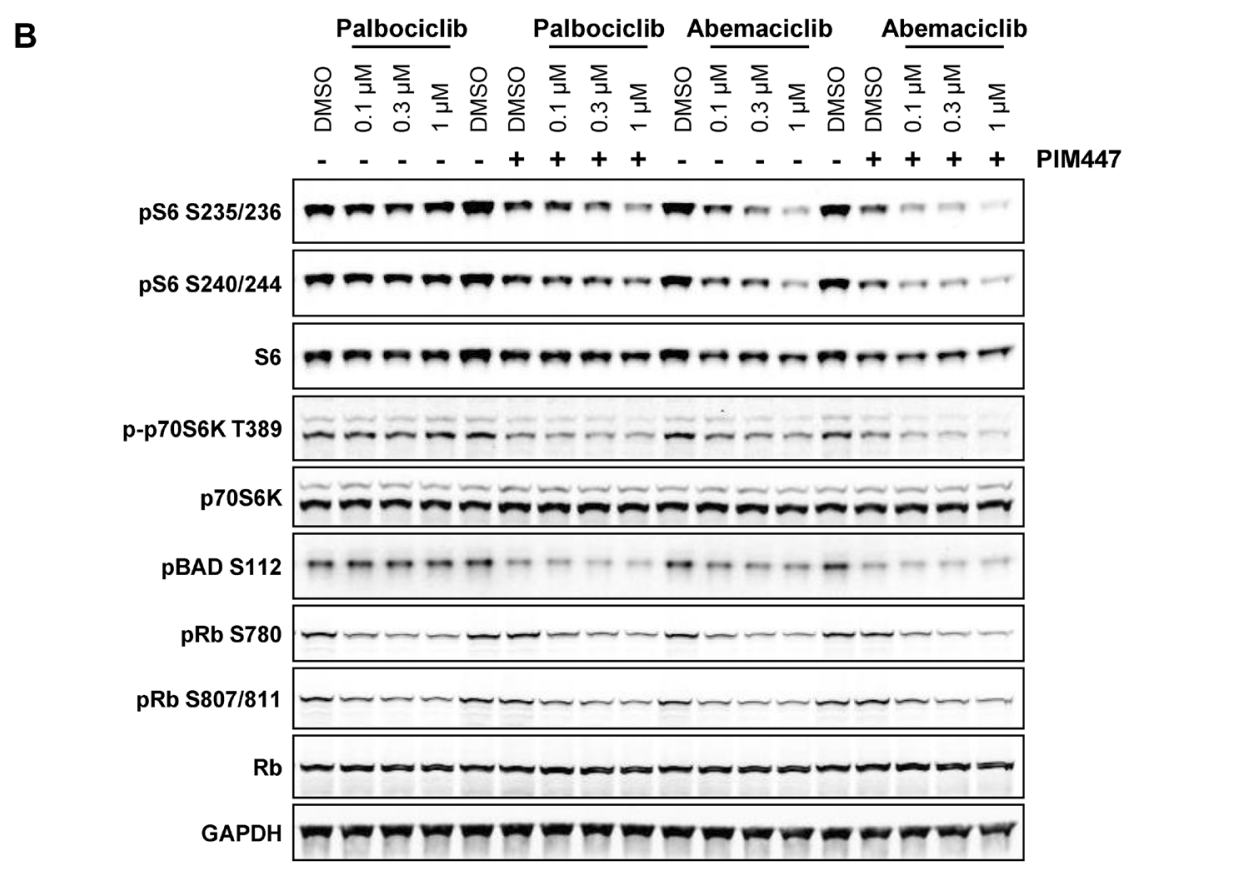

Figure 3: Combination CDK4/6 and PIM kinase inhibition suppresses mTOR signaling and cell growth. (A) DMS-53 cells were treated with the combination of PIM inhibitor PIM447 and abemaciclib or palbociclib for 2DT and cell growth was assessed by CellTiter-Glo. Curve shift analysis [66] was used to calculate a combination index (CI) as an indication of additivity or synergy between the compounds. (B) DMS-53 cells were treated with the combination of PIM inhibitor PIM447 (0.3 $\mu \mathrm{M})$ and abemaciclib or palbociclib for $4 \mathrm{~h}$ and analyzed by western blot. 
further investigation. S6 phosphorylation was shown to be temporally regulated during cell cycle progression, with lower levels of phosphorylation observed in G1 [43]. Negative regulation of TSC 2 by exogenous cyclin D/CDK4/6 has been reported [13], and CDK4 or cyclin D1 knockdown led to a modest suppression of mTOR signaling in HER2+ breast cancer cells [9], effects which may contribute to the observed subtle reduction in S6 phosphorylation following long-term treatment with palbociclib (Supplementary Figure $1 F$ ) or CDK4/6 knockdown (Figure 1D). Conversely, longterm treatment of pancreatic cancer cells with palbociclib actually resulted in upregulation of mTOR signaling [44], and $\mathrm{Rb}$ loss or CDK4/ 6 inhibition activated mTORC2 and Akt in ovarian cancer, TNBC, and osteosarcoma cells [45], suggesting that the impact of CDK4/6 on mTOR signaling may vary by tumor type or genetic background.

Interpretation of in vitro biochemical and cellular assays evaluating targets of kinase inhibitors and translation to potential in vivo and clinical effects requires consideration of activities achievable at biologically relevant ATP concentrations and clinically relevant drug concentrations. For example, while abemaciclib displayed inhibitory activity against CDK9 in biochemical kinase assays ( $\mathrm{IC}_{50} 57 \pm 42 \mathrm{nM}$ ) [20], this activity did not translate to inhibition of CDK9 substrates in cells at circulating steady-state drug concentrations achieved in patients, or even at concentrations up to $20 \mu \mathrm{M}$ $[2,46]$. Inhibition of PIM kinases by abemaciclib was also identified through biochemical assays and was validated through the analysis of the PIM substrate BAD in cells [20]. In the present study, inhibition of mTOR signaling by abemaciclib was observed at concentrations $<1 \mu \mathrm{M}$ in cells, which falls within the clinical exposure levels for the drug and its active metabolites [28], and thus is predicted to be potentially clinically relevant. Although clinical samples were not available to directly measure
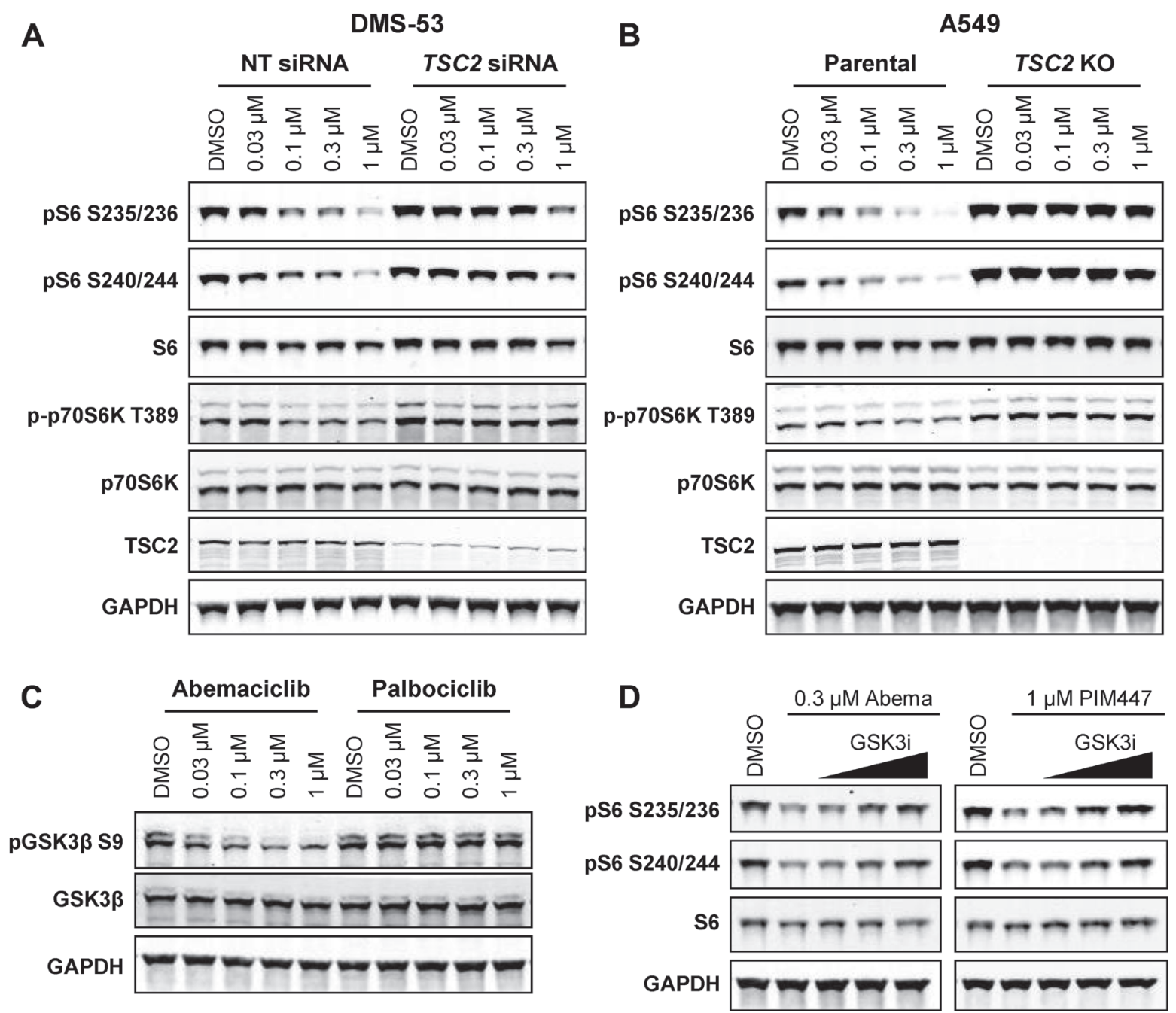

Figure 4: Inhibition of mTOR signaling by abemaciclib requires TSC2 and GSK3ß. (A) DMS-53 cells were transfected with TSC2 or non-targeting control (NT) siRNA for $48 \mathrm{~h}$ prior to treatment with abemaciclib for $4 \mathrm{~h}$ and analysis by western blot. (B) A549 parental or TSC2 KO cells were treated and analyzed as in A. (C) DMS-53 cells were treated with the indicated concentrations of abemaciclib or palbociclib for $4 \mathrm{~h}$ and analyzed by western blot. (D) DMS-53 cells were treated with abemaciclib or PIM447, alone or in combination with increasing concentrations of GSK3 inhibitor LY2090314 $(0.005,0.05$, or $0.5 \mu \mathrm{M})$, for $4 \mathrm{~h}$ and analyzed by western blot. 
protein markers of pathway activation, such as S6 phosphorylation, analysis of the $\mathrm{mTORC} 1$ gene signature in RNAseq data from a phase 2 study of neoadjuvant abemaciclib (neoMONARCH, NCT02441946) revealed that abemaciclib monotherapy did indeed inhibit the mTOR pathway in $\mathrm{HR}+$ breast cancer patients.

Though much work on PIM kinases has focused on their roles in hematological malignancies, with

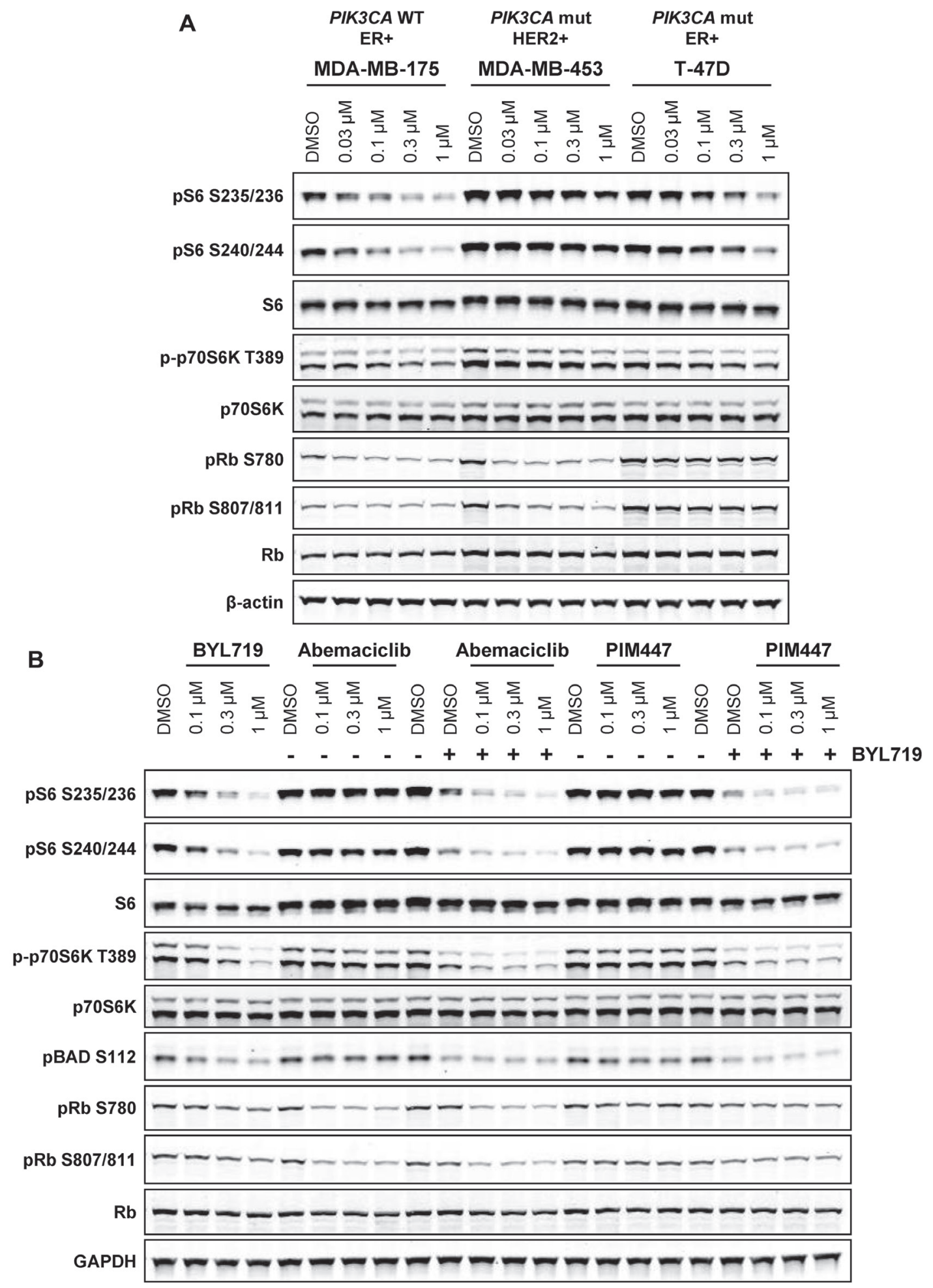

Figure 5: PI3K activity compensates for PIM inhibition in PIK3CA mutant breast cancer. (A) MDA-MB-175 (PIK3CA wt), MDA-MB-453 (PIK3CA mutant, HER2+), and T-47D (PIK3CA mutant) cells were treated with the indicated concentrations of abemaciclib for $4 \mathrm{~h}$ and analyzed by western blot. (B) MDA-MB-453 cells were treated with the combination of PI3K inhibitor BYL719 (0.3 $\mu \mathrm{M})$ and abemaciclib or PIM447 for $4 \mathrm{~h}$ and analyzed by western blot. 
clinical testing of PIM inhibitors as monotherapy in multiple myeloma, acute myeloid leukemia, malignant lymphoma, and non-Hodgkin's lymphoma [47], they have also been shown to be overexpressed in multiple solid tumor types, including breast, prostate, pancreatic, gastric, hepatocellular, and colorectal cancers [48-50] and hypothesized to contribute to disease progression in many cases. Across a large cell panel, the activity profiles of abemaciclib and palbociclib were closely related ( $p<0.001$ ), and $R B 1$ depletion conferred resistance to abemaciclib, suggesting that the predominant anti-tumor activity is driven by CDK4/6 inhibition [2]. Additionally, PIM447 was largely inactive as monotherapy across the cell panel (data not shown), suggesting that PIM inhibition alone will not confer significant anti-tumor activity. However, we note that in our prior study [2], there were a
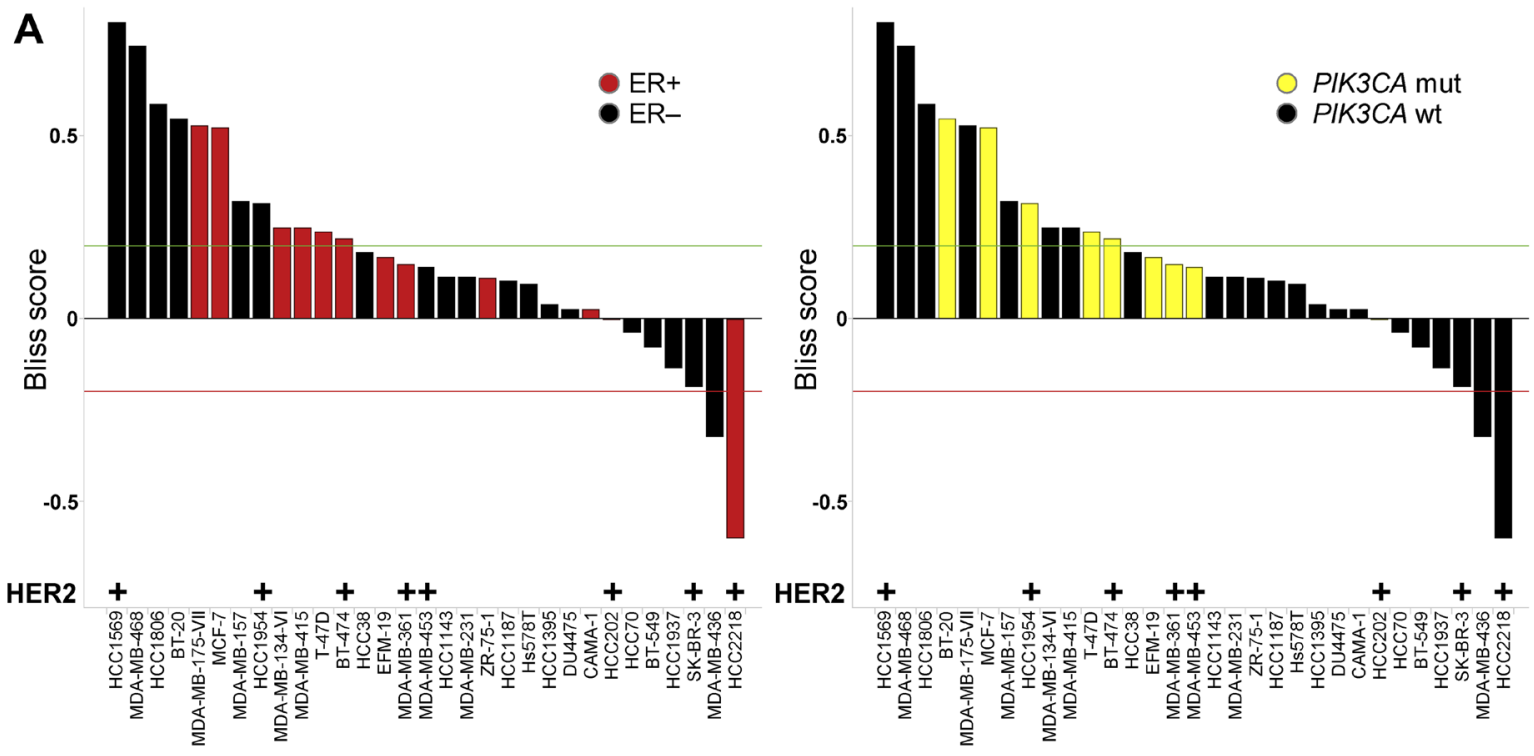

B
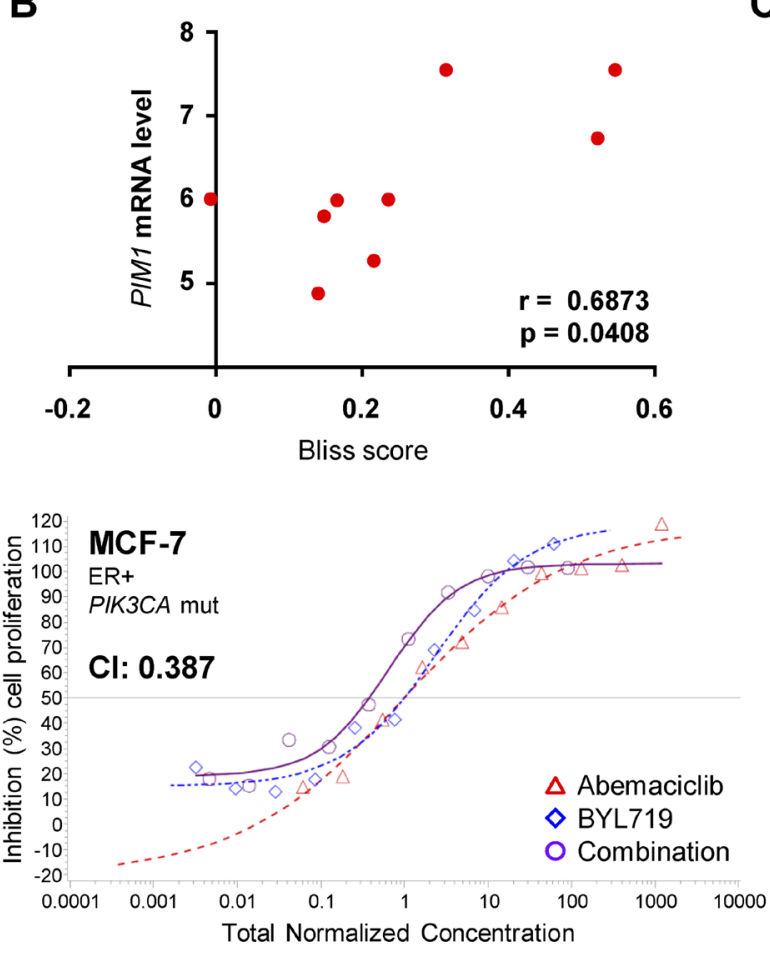

C
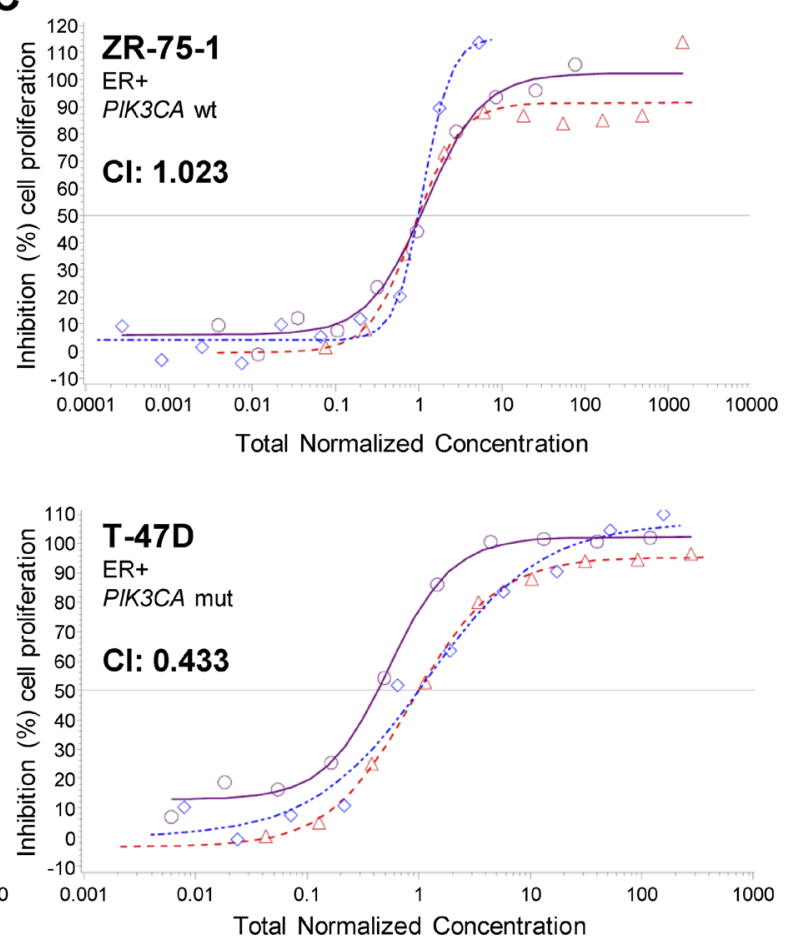

Figure 6: Combination treatment with abemaciclib and BYL719 synergistically inhibits breast cancer cell growth. (A) A panel of 31 breast cancer cell lines was treated with the combination of abemaciclib and PI3K inhibitor BYL719 for 2DT and cell growth was assessed by PI staining. ER expression/PIK3CA mutation status are displayed. (B) Correlation of PIMI mRNA levels (Cancer Cell Line Encyclopedia (CCLE) [68]) and abemaciclib-BYL719 Bliss score. (C) Confirmatory assays of MCF-7 (PIK3CA mutant), T-47D (PIK3CA mutant), or ZR-75-1 (PIK3CA wt) cells treated with the combination of abemaciclib and BYL719 for 2DT and assessed by PI staining. Curve shift analysis was used to calculate a combination index (CI) as an indication of additivity or synergy between the compounds. 
number of cancer cell lines in which the anti-proliferative $\mathrm{IC}_{50}$ of abemaciclib was at least $5 \times$ lower than palbociclib. Intriguingly, several of these, including MDA-MB-157 and HCC1143, are from triple negative breast cancer, which has been reported to depend on PIM kinase [51, 52]. Further work is necessary to determine whether PIM inhibition contributes to the diverging activity of the two drugs in these, or other, contexts and, more generally, to investigate the cancer types and context where combined CDK4/6 and PIM inhibition may be advantageous. In additional to their roles in cell growth and proliferation, PIM kinases have also been hypothesized to have immunosuppressive functions in cancer [53], in part via inhibition of JAK-STAT signaling $[54,55]$. Whether these effects contribute to the ability of abemaciclib to promote anti-tumor immunity [56] remains to be evaluated.

PIM and CDK4/6 have each been implicated in resistance to PI3K inhibition [6, 15], while PI3K signaling can also contribute to CDK4/6 inhibitor resistance [57]. This crosstalk and the convergence of PIM and Akt on mTOR signaling led us to test the combination of BYL719 and abemaciclib in breast cancer cells, where we observed synergistic inhibition of cell growth and attenuation of downstream signaling. Dual inhibition of CDK4/6 and PIM by abemaciclib could present an advantage in combination with PI3K pathway inhibitors to suppress signaling and combat potential mechanisms of resistance to inhibitors of both PI3K and CDK4/6. PIM kinases have also been identified as mediators of drug resistance in other contexts [58], including resistance to MET inhibitors in lung or gastric cancers $[59,60]$, suggesting the application of PIM inhibition to other therapeutic combinations in additional tumor indications.

Several beneficial combinations of CDK4/6 inhibitors with compounds targeting PI3K/Akt/mTOR signaling have been reported in the preclinical setting $[6,7,40,57,61-65]$. Meanwhile, combinations of CDK4/6 inhibitors with $\mathrm{PI} 3 \mathrm{~K} / \mathrm{mTOR}$ pathway inhibitors are at various stages of clinical testing, including abemaciclib with PI3K/mTOR inhibitor LY3023414 or everolimus; palbociclib with $\mathrm{PI} 3 \mathrm{~K} / \mathrm{mTOR}$ inhibitor PF-05212384, PI3K inhibitors GDC-0077, taselisib, or pictilisib, or everolimus; and ribociclib with PI3K inhibitors alpelisib or buparlisib, or everolimus [18]. Ultimately, these studies will provide important information to understand the best strategies for combination therapy to take advantage of these complex biological relationships to improve clinical efficacy and circumvent resistance mechanisms.

\section{MATERIALS AND METHODS}

\section{Cell lines and compounds}

A549, BT-20, BT-474, BT-549, CAMA-1, DMS-53, DU4475, HCC38, HCC70, HCC202, HCC1143, HCC1187, HCC1395, HCC1569, HCC1806, HCC1937, HCC1954,

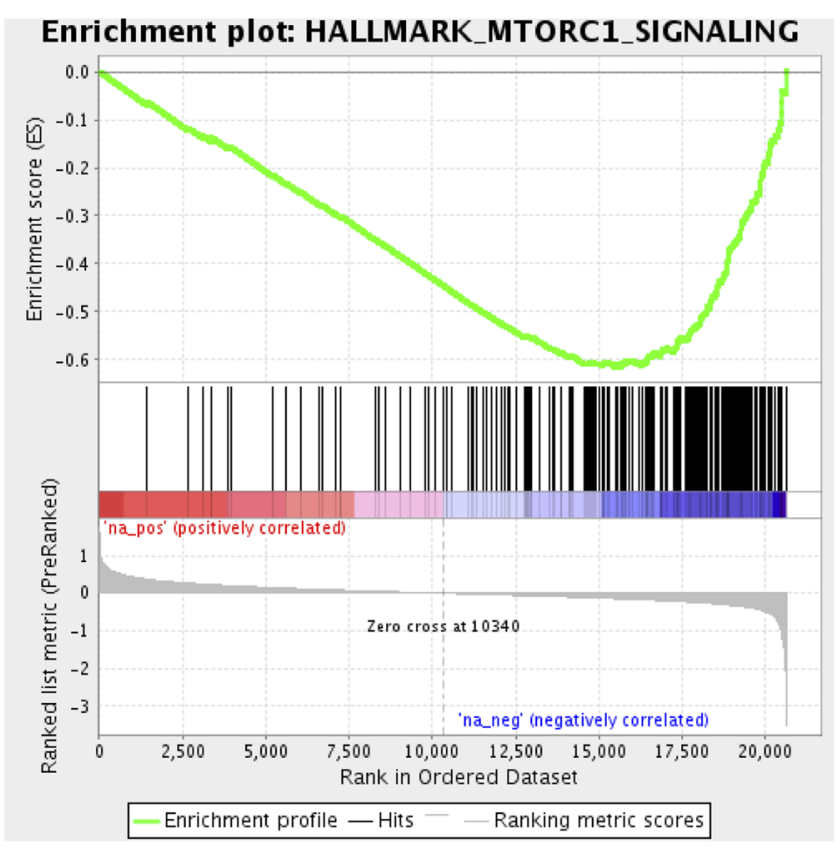

\section{ES -0.61 \\ NES -2.71}

Figure 7: Suppression of mTOR signaling in breast cancer patients treated with abemaciclib. GSEA enrichment plot for mTOR signature following two weeks of abemaciclib monotherapy treatment (early vs baseline) in the neoMONARCH clinical trial. Nominal $p<0.01$, FDR $q<0.01$. ES, enrichment score; NES, normalized enrichment score; FDR, false discovery rate. 
HCC2218, HCT-116, Hs578T, Jeko-1, MCF-7, MDAMB-134-VI, MDA-MB-157, MDA-MB-175-VII, MDAMB-231, MDA-MB-361, MDA-MB-415, MDA-MB-436, MDA-MB-453, MDA-MB-468, MiaPaCa2, SK-BR-3, NCI-H441, SK-MEL-28, T-47D, U2OS, and ZR-75-1 were obtained from the American Type Culture Collection (ATCC) and cultured according to vendor recommendations. EFM-19 were obtained from the German Collection of Microorganisms and Cell Cultures (DSMZ) and SNU-886 were from the Korean Cell Line Bank (KCLB).

Abemaciclib, palbociclib, ribociclib, PIM447, BYL719, LY2090314, everolimus, DYRK1Bi AZ cpd 33 [32], dinaciclib, GSK2334470, abemaciclib metabolites M2 and M20 [28], and additional CDK4/6i (see Figure 2C [33],) were synthesized by Lilly Research Laboratories. AZD1208 (S7104) and additional palbociclib (S1579, see Supplementary Figure 1A) were purchased from Selleck Chemicals.

\section{Western blot analysis}

Cells were treated as indicated and western blot analysis was performed as previously described [2]. The pS6 S235/236 (4858), pS6 S240/244 (2215), S6 (2317), 4EBP1 (9644), pRb S780 (8180), pRb S807/811 (8516), Rb (9309), pBAD S112 (5284), p-p70S6K T389 (9205), p70S6K (2708), PIM1 (3247), PIM2 (4730), PIM3 (4165), TSC2 (4308), pGSK3 $\beta$ S9 (5558), GSK3 $\beta$ (9315), pPRAS40 T246 (2997), PRAS40 (2691), and GAPDH (2118) primary antibodies were purchased from Cell Signaling Technology, while the CDK4 (ab75511) and CDK6 (ab124821) antibodies were from Abcam and $\beta$-actin (A5441) was from Sigma-Aldrich. IRDye secondary antibodies were from LI-COR.

\section{Cell growth assays}

Cells were plated in 96-well plates and allowed to attach overnight prior to treatment with increasing concentrations of the indicated compounds. Cell growth was assessed by CellTiter-Glo ${ }^{\circledR}$ Luminescent Cell Viability Assay (CTG; Promega), according to manufacturer's instructions, or PI staining following treatment for two cell doubling times (2DT). PI staining was performed as previously described [46]. Response to drug combinations was evaluated by curve shift analysis [66] or Bliss independence analysis [67].

\section{In vivo study}

All animal studies were performed in accordance with American Association for Laboratory Animal Care institutional guidelines, and all protocols were approved by the Eli Lilly and Company Animal Care and Use Committee. A549 cells $\left(5 \times 10^{6}\right)$ were suspended in a 1:1 mixture of HBSS and Matrigel (BD Biosciences) and injected subcutaneously into the rear flank of female CB17SCID mice (Envigo). Mice were subsequently randomized into treatment groups ( $n=5$ /group) based on tumor volume and body weight when mean tumor volume reached $200-250 \mathrm{~mm}^{3}$. A single dose $(50 \mathrm{mg} /$ $\mathrm{kg}$ ) of abemaciclib or palbociclib was administered by oral gavage (PO) with tumors collected 2, 4, 8, or $24 \mathrm{~h}$ post-treatment. In vivo doses were selected in part based on previous pharmacokinetic data (data not shown), with $50 \mathrm{mg} / \mathrm{kg}$ palbociclib in mice corresponding to $\sim 3 \times$ clinical exposures at $125 \mathrm{mg}$ QD dose. Abemaciclib was formulated in $1 \%$ hydroxyethyl cellulose (HEC, Natrosol; Ashland) in $25 \mathrm{mM}$ phosphate buffer, $\mathrm{pH} 2$, while palbociclib was formulated in 5\% N-methyl-2pyrrolidone (NMP; Sigma-Aldrich), $0.05 \%$ antifoam in $25 \mathrm{mM}$ phosphate buffer, $\mathrm{pH}$ 2. Vehicle $1 \%$ HEC in 25 $\mathrm{mM}$ phosphate buffer served as control. Tumors were homogenized and lysates were analyzed by western blot as described above.

\section{RNAi}

Cells plated in 6-well plates were transfected with 25 nM CDK4 (L-003238-00-0005), CDK6 (L-00324000-0005), PIM1 (L-003923-00-0005), PIM2 (L-00535900-0005), PIM3 (L-032287-00-0005), TSC2 (L-00302900-0005), or non-targeting control (D-001810-10-05) SMARTpool siRNA (Dharmacon) using DharmaFECT1 transfection reagent (T-2001-01, Dharmacon). Following $48 \mathrm{~h}$ transfection, cells were treated as indicated and/or lysed and analyzed by western blot.

\section{CRISPR RB1 and TSC2 knockout}

DMS-53 and A549 cells were transfected with a plasmid containing Cas9 and RB1- or TSC2specific sgRNAs, respectively, and subjected to puromycin selection (HD Biosciences, Shanghai, China). $R B 1, \quad 5^{\prime}$ GGGTCCGGGGGTTCCGCGG 3'; TSC2, 5' CTGTCGCACCATCAACGTCA 3'; 5' CTGCAACTACCACGCTGCT 3' (Sangon Biotech). For $R B 1$, pooled $\mathrm{KO}$ cells were additionally subjected to selection with $2 \mu \mathrm{M}$ palbociclib and $R B 1 \mathrm{KO}$ was confirmed by western blot. For TSC2, single clones were isolated and TSC2 KO was validated by Sanger sequencing.

\section{Structural modeling}

Preparation of the in-house PIM1 proteinligand complex x-ray, the glide docking model and the superposition of palbociclib on top of abemaciclib were performed using the available Protein Preparation Wizard, Glide and manual atom pairs superposition panels of Maestro version 12.0.12 (Schrodinger Suites Release 2019-2). 
An in-house PIM1 protein-ligand complex crystal structure where the ligand was structurally similar to abemaciclib (benzimidazole ring and fluorine atoms and their positions were conserved) was prepared. Waters were removed and not considered. A glide grid centered in the ligand and with defined two constrains to Glu121 (aromatic hydrogen bond) and Lys67 (H-bond) was built. This grid was used to dock abemaciclib 3D structure (taken from a published crystal structure of abemaciclib in CDK6, 5L2S. pdb) [24] using XP (Extra-precision) and rigid ligand docking (no sampling). The binding docking pose satisfied both constrains. The highest scored pose was selected and used to atom-pair superimpose palbociclib 3D structure (taken from 5L2I. pdb) [24] on top of it. The atom pairs list used was built based on the comparison between abemaciclib and palbociclib in previously cited crystal structures bound to CDK6.

\section{Clinical samples and GSEA}

Postmenopausal early stage (Stage I [tumor $\geq 1 \mathrm{~cm}$ ], II, IIIA or IIIB) HR+, HER2- breast cancer patients were randomized to one of three treatment arms: abemaciclib monotherapy (150 mg orally every 12 hours), anastrozole monotherapy (1 mg orally once daily), or abemaciclib in combination with anastrozole for two weeks, followed by an additional fourteen weeks of treatment with combination abemaciclib and anastrozole. Biopsies were obtained at baseline, following two weeks of initial therapy, and following the additional fourteen weeks of combination therapy. The study was conducted in compliance with the principles of good clinical practice, applicable laws and regulations, and the Declaration of Helsinki. The protocol was approved by each institution's review board, and written informed consent was collected from patients before enrollment. This study is registered with ClinicalTrials. gov (NCT02441946). Extracted RNA from FFPE biopsy samples was analyzed by Illumina Truseq RNA exome RNA sequencing (RNAseq) and GSEA as previously described [41].

\section{Abbreviations}

CDK: cyclin-dependent kinase; SCLC: small cell lung cancer; MCL: mantle cell lymphoma; NSCLC: non-small cell lung cancer; TNBC: triple negative breast cancer; GSK3 $\beta$ : glycogen synthase kinase $3 \beta$; GSEA: gene set enrichment analysis; CTG: CellTiter-Glo ${ }^{\circledR}$ Luminescent Cell Viability Assay.

\section{Author contributions}

LML: data acquisition and analysis/interpretation, manuscript drafting and revision; KB: data acquisition and analysis/interpretation, manuscript revision; MB: data analysis/interpretation; CM: data acquisition; $\mathrm{CB}$ : data acquisition; JRS: data acquisition; JMS: data acquisition and analysis/interpretation; SMG: data acquisition and analysis/ interpretation; AMM: experimental design; XSY: experimental design; WW: data acquisition; MJL: experimental design; $\mathrm{XG}$ : data interpretation; FFM: conception and design; VMJ: data acquisition and interpretation; SGB: conception and design, data interpretation, manuscript revision.

\section{CONFLICTS OF INTEREST}

All authors are current or former employees and shareholders of Eli Lilly and Company.

\section{FUNDING}

This work was supported by Eli Lilly and Company.

\section{REFERENCES}

1. O'Leary B, Finn RS, Turner NC. Treating cancer with selective CDK4/6 inhibitors. Nat Rev Clin Oncol. 2016; 13:417-430. https://doi.org/10.1038/nrclinonc.2016.26. [PubMed]

2. Gong X, Litchfield LM, Webster Y, Chio LC, Wong SS, Stewart TR, Dowless M, Dempsey J, Zeng Y, Torres R, Boehnke K, Mur C, Marugán C, et al. Genomic Aberrations that Activate D-type Cyclins Are Associated with Enhanced Sensitivity to the CDK4 and CDK6 Inhibitor Abemaciclib. Cancer Cell. 2017; 32:761-76.e6. https://doi.org/10.1016/j. ccell.2017.11.006. [ $\underline{\text { PubMed] }}$

3. Qie S, Diehl JA. Cyclin D1, cancer progression, and opportunities in cancer treatment. J Mol Med (Berl). 2016; 94:1313-1326. https://doi.org/10.1007/s00109-016-1475-3. [PubMed]

4. Musgrove EA, Caldon CE, Barraclough J, Stone A, Sutherland RL. Cyclin D as a therapeutic target in cancer. Nat Rev Cancer. 2011; 11:558-572. https://doi.org/10.1038/nrc3090. [PubMed]

5. Klein EA, Assoian RK. Transcriptional regulation of the cyclin D1 gene at a glance. J Cell Sci. 2008; 121:38533857. https://doi.org/10.1242/jcs.039131. [PubMed]

6. Vora Sadhna R, Juric D, Kim N, Mino-Kenudson M, Huynh T, Costa C, Lockerman Elizabeth L, Pollack Sarah F, Liu M, Li X, Lehar J, Wiesmann M, Wartmann M, et al. CDK 4/6 Inhibitors Sensitize PIK3CA Mutant Breast Cancer to PI3K Inhibitors. Cancer Cell. 2014; 26:136-149. https://doi. org/10.1016/j.ccr.2014.05.020. [ [PubMed]

7. Heilmann AM, Perera RM, Ecker V, Nicolay BN, Bardeesy N, Benes CH, Dyson NJ. CDK4/6 and IGF1 Receptor Inhibitors Synergize to Suppress the Growth of p16 INK4A-Deficient Pancreatic Cancers. Cancer Res. 2014; 74:3947-3958. https:// doi.org/10.1158/0008-5472.CAN-13-2923. [PubMed]

8. Corcoran RB, Rothenberg SM, Hata AN, Faber AC, Piris A, Nazarian RM, Brown RD, Godfrey JT, Winokur D, Walsh J, Mino-Kenudson M, Maheswaran S, Settleman $\mathrm{J}$, et al. TORC1 suppression predicts responsiveness to RAF and MEK inhibition in BRAF-mutant melanoma. 
Sci Transl Med. 2013; 5:196ra98. https://doi.org/10.1126/ scitranslmed.3005753. [PubMed]

9. Goel S, Wang Q, Watt AC, Tolaney SM, Dillon DA, Li W, Ramm S, Palmer AC, Yuzugullu H, Varadan V, Tuck D, Harris LN, Wong KK, et al. Overcoming Therapeutic Resistance in HER2-Positive Breast Cancers with CDK4/6 Inhibitors. Cancer Cell. 2016; 29:255-269. https://doi. org/10.1016/j.ccell.2016.02.006. [PubMed]

10. $\mathrm{Yu}$ J, Henske EP. Estrogen-Induced Activation of Mammalian Target of Rapamycin Is Mediated via Tuberin and the Small GTPase Ras Homologue Enriched in Brain. Cancer Res. 2006; 66:9461-9466. https://doi. org/10.1158/0008-5472.CAN-06-1895. [PubMed]

11. Boulay A, Rudloff J, Ye J, Zumstein-Mecker S, O'Reilly T, Evans DB, Chen S, Lane HA. Dual Inhibition of mTOR and Estrogen Receptor Signaling In vitro Induces Cell Death in Models of Breast Cancer. Clin Cancer Res. 2005; 11:5319 5328. https://doi.org/10.1158/1078-0432.CCR-04-2402. [PubMed]

12. Michaloglou C, Crafter C, Siersbæk R, Delpuech O, Curwen JO, Carnevalli LS, Staniszewska AD, Polanska UM, Cheraghchi-Bashi A, Lawson M, Chernukhin I, McEwen $\mathrm{R}$, Carroll JS, et al. Combined inhibition of mTOR and CDK4/6 is required for optimal blockade of E2F function and long term growth inhibition in estrogen receptor positive breast cancer. Mol Cancer Ther. 2018; 17:908-920. https:// doi.org/10.1158/1535-7163.MCT-17-0537. [PubMed]

13. Zacharek SJ, Xiong Y, Shumway SD. Negative regulation of TSC1-TSC2 by mammalian D-type cyclins. Cancer Res. 2005; 65:11354-11360. https://doi.org/10.1158/0008-5472. CAN-05-2236. [PubMed]

14. Romero-Pozuelo J, Demetriades C, Schroeder P, Teleman AA. CycD/Cdk4 and Discontinuities in Dpp Signaling Activate TORC1 in the Drosophila Wing Disc. Dev Cell. 2017; 42:376-87.e5. https://doi.org/10.1016/j. devcel.2017.07.019. [PubMed]

15. Le X, Antony R, Razavi P, Treacy DJ, Luo F, Ghandi M, Castel P, Scaltriti M, Baselga J, Garraway LA. Systematic Functional Characterization of Resistance to PI3K Inhibition in Breast Cancer. Cancer Discov. 2016; 6:1134-1147. https:// doi.org/10.1158/2159-8290.CD-16-0305. [PubMed]

16. Warfel NA, Kraft AS. PIM kinase (and Akt) biology and signaling in tumors. Pharmacol Ther. 2015; 151:41-49. https://doi.org/10.1016/j.pharmthera.2015.03.001. [PubMed]

17. Turner NC, Neven P, Loibl S, Andre F. Advances in the treatment of advanced oestrogen-receptor-positive breast cancer. Lancet. 2017; 389:2403-2414. https://doi. org/10.1016/S0140-6736(16)32419-9. [PubMed]

18. Ingham M, Schwartz GK. Cell-Cycle Therapeutics Come of Age. J Clin Oncol. 2017; 35:2949-2959. https://doi. org/10.1200/JCO.2016.69.0032. [PubMed]

19. Ramos-Esquivel A, Hernández-Steller H, Savard MF, Landaverde DU. Cyclin-dependent kinase 4/6 inhibitors as first-line treatment for post-menopausal metastatic hormone receptor-positive breast cancer patients: a systematic review and meta-analysis of phase III randomized clinical trials.
Breast Cancer. 2018; 25:479-488. https://doi.org/10.1007/ s12282-018-0848-6. [PubMed]

20. Gelbert LM, Cai S, Lin X, Sanchez-Martinez C, del Prado M, Lallena MJ, Torres R, Ajamie RT, Wishart GN, Flack RS, Neubauer BL, Young J, Chan EM, et al. Preclinical characterization of the CDK4/6 inhibitor LY2835219: in-vivo cell cycle-dependent/independent anti-tumor activities alone/ in combination with gemcitabine. Invest New Drugs. 2014; 32:825-837. https://doi.org/10.1007/s10637-014-0120-7. [PubMed]

21. Patnaik A, Rosen LS, Tolaney SM, Tolcher AW, Goldman JW, Gandhi L, Papadopoulos KP, Beeram M, Rasco DW, Hilton JF, Nasir A, Beckmann RP, Schade AE, et al. Efficacy and Safety of Abemaciclib, an Inhibitor of CDK4 and CDK6, for Patients with Breast Cancer, Non-Small Cell Lung Cancer, and Other Solid Tumors. Cancer Discov. 2016; 6:740-53. https://doi.org/10.1158/2159-8290.CD-160095. [PubMed]

22. Dickler MN, Tolaney SM, Rugo HS, Cortés J, Diéras V, Patt D, Wildiers H, Hudis CA, O’Shaughnessy J, Zamora E, Yardley DA, Frenzel M, Koustenis A, et al. MONARCH 1, A Phase II Study of Abemaciclib, a CDK4 and CDK6 Inhibitor, as a Single Agent, in Patients with Refractory HR+/HER2- Metastatic Breast Cancer. Clin Cancer Res. 2017; 23:5218-5224. https:// doi.org/10.1158/1078-0432.CCR-17-0754. [PubMed]

23. Verzenio [package insert]. Indianapolis, IN: Eli Lilly and Company; 2019. https://www.accessdata.fda.gov/drugsatfda docs/label/2019/208716s0031bl.pdf.

24. Chen P, Lee NV, Hu W, Xu M, Ferre RA, Lam H, Bergqvist S, Solowiej J, Diehl W, He YA, Yu X, Nagata A, VanArsdale T, et al. Spectrum and Degree of CDK Drug Interactions Predicts Clinical Performance. Mol Cancer Ther. 2016; 15:2273-2281. https://doi.org/10.1158/1535-7163.MCT-16-0300. [PubMed]

25. Knudsen ES, Hutcheson J, Vail P, Witkiewicz AK. Biological specificity of CDK4/6 inhibitors: dose response relationship, in vivo signaling, and composite response signature. Oncotarget. 2017; 8:43678-43691. https://doi. org/10.18632/oncotarget.18435. [ [PubMed]

26. Hafner M, Mills CE, Subramanian K, Chen C, Chung M, Boswell SA, Everley RA, Liu C, Walmsley CS, Juric D, Sorger PK. Multiomics Profiling Establishes the Polypharmacology of FDA-Approved CDK4/6 Inhibitors and the Potential for Differential Clinical Activity. Cell Chem Biol. 2019; 26:1067-1080.e8. https://doi. org/10.1016/i.chembiol.2019.05.005. [ubMed]

27. Cousins EM, Goldfarb D, Yan F, Roques J, Darr DB, Johnson GL, Major MB. Competitive Kinase Enrichment Proteomics Reveals that Abemaciclib Inhibits GSK3 $\beta$ and Activates WNT Signaling. Mol Cancer Res. 2018; 16:333-344. https:// doi.org/10.1158/1541-7786.MCR-17-0468. [PubMed]

28. Kulanthaivel P, Mahadevan D, Turner PK, Royalty J, Ng WT, Yi P, Rehmel J, Cassidy K, Chappell J. Abstract CT153: Pharmacokinetic drug interactions between abemaciclib and CYP3A inducers and inhibitors. Cancer Res. 2016; 76:CT153. https://doi.org/10.1158/1538-7445. AM2016-CT153. 
29. Burke T, Torres R, McNulty A, Dempsey J, Kolis S, Kulanthaivel P, Beckmann R. Abstract 2830: The major human metabolites of abemaciclib are inhibitors of CDK4 and CDK6. Cancer Res. 2016; 76:2830. https://doi.org/10.1158/15387445.AM2016-2830.

30. Burger MT, Nishiguchi G, Han W, Lan J, Simmons R, Atallah G, Ding Y, Tamez V, Zhang Y, Mathur M, Muller $\mathrm{K}$, Bellamacina $\mathrm{C}$, Lindvall $\mathrm{MK}$, et al. Identification of N-(4-((1R,3S,5S)-3-Amino-5-methylcyclohexyl)pyridin-3yl)-6-(2,6-difluorophenyl)- 5-fluoropicolinamide (PIM447), a Potent and Selective Proviral Insertion Site of Moloney Murine Leukemia (PIM) 1, 2, and 3 Kinase Inhibitor in Clinical Trials for Hematological Malignancies. J Med Chem. 2015; 58:8373-8386. https://doi.org/10.1021/acs. jmedchem.5b01275. [PubMed]

31. Keeton EK, McEachern K, Dillman KS, Palakurthi S, Cao Y, Grondine MR, Kaur S, Wang S, Chen Y, Wu A, Shen M, Gibbons FD, Lamb ML, et al. AZD1208, a potent and selective pan-Pim kinase inhibitor, demonstrates efficacy in preclinical models of acute myeloid leukemia. Blood. 2014; 123:905-913. https://doi.org/10.1182/blood-2013-04-495366. [PubMed]

32. Kettle JG, Ballard P, Bardelle C, Cockerill M, Colclough N, Critchlow SE, Debreczeni J, Fairley G, Fillery S, Graham MA, Goodwin L, Guichard S, Hudson K, et al. Discovery and Optimization of a Novel Series of Dyrk1B Kinase Inhibitors To Explore a MEK Resistance Hypothesis. J Med Chem. 2015; 58:2834-2844. https://doi.org/10.1021/ acs.jmedchem.5b00098. [PubMed]

33. Coates DA, Gelbert LM, Knobeloch JM, De Dios Magana A, De Prado Gonzalez A, Filadelfa Del Prado Catalina M, Garcia Paredes MC, Martin De La Nava EM, Martin Ortega Finger MD, Martinez Perez JA, Mateo Herranz AI, Perez Martinez C, Sanchez Martinez C, inventors; Eli Lilly and Co, assignee. Protein kinase inhibitors. United States patent US 7855211. 2010 Dec 21.

34. Zhang F, Beharry ZM, Harris TE, Lilly MB, Smith CD, Mahajan S, Kraft AS. PIM1 Protein Kinase regulates PRAS40 phosphorylation and mTOR activity in FDCP1 cells. Cancer Biol Ther. 2009; 8:846-853. https://doi. org/10.4161/cbt.8.9.8210. [PubMed]

35. Lu J, Zavorotinskaya T, Dai Y, Niu XH, Castillo J, Sim J, Yu J, Wang Y, Langowski JL, Holash J, Shannon K, Garcia PD. Pim2 is required for maintaining multiple myeloma cell growth through modulating TSC2 phosphorylation. Blood. 2013; 122:1610-1620. https://doi.org/10.1182/blood-2013-01-481457. [PubMed]

36. Santio NM, Salmela M, Arola H, Eerola SK, Heino J, Rainio EM, Koskinen PJ. The PIM1 kinase promotes prostate cancer cell migration and adhesion via multiple signalling pathways. Exp Cell Res. 2016; 342:113-124. https://doi.org/10.1016/j.yexcr.2016.02.018. [PubMed]

37. Inoki $\mathrm{K}$, Ouyang $\mathrm{H}$, Zhu $\mathrm{T}$, Lindvall $\mathrm{C}$, Wang $\mathrm{Y}$, Zhang $\mathrm{X}$, Yang Q, Bennett C, Harada Y, Stankunas K, Wang CY, He $\mathrm{X}$, MacDougald OA, et al. TSC2 integrates Wnt and energy signals via a coordinated phosphorylation by AMPK and GSK3 to regulate cell growth. Cell. 2006; 126:955-968. https://doi.org/10.1016/j.cell.2006.06.055. [PubMed]
38. Atkinson JM, Rank KB, Zeng Y, Capen A, Yadav V, Manro JR, Engler TA, Chedid M. Activating the Wnt/ $\beta$-Catenin Pathway for the Treatment of Melanoma - Application of LY2090314, a Novel Selective Inhibitor of Glycogen Synthase Kinase-3. PLoS One. 2015; 10:e0125028. https:// doi.org/10.1371/journal.pone.0125028. [PubMed]

39. Moody SE, Schinzel AC, Singh S, Izzo F, Strickland MR, Luo L, Thomas SR, Boehm JS, Kim SY, Wang ZC, Hahn WC. PRKACA mediates resistance to HER2-targeted therapy in breast cancer cells and restores anti-apoptotic signaling. Oncogene. 2015; 34:2061-2071. https://doi. org/10.1038/onc.2014.153. [PubMed]

40. Jansen VM, Bhola NE, Bauer JA, Formisano L, Lee KM, Hutchinson KE, Witkiewicz AK, Moore PD, Estrada MV, Sanchez V, Ericsson PG, Sanders ME, Pohlmann PR, et al. Kinome-Wide RNA Interference Screen Reveals a Role for PDK1 in Acquired Resistance to CDK4/6 Inhibition in ERPositive Breast Cancer. Cancer Res. 2017; 77:2488-2499. https://doi.org/10.1158/0008-5472.CAN-16-2653. [PubMed]

41. Hurvitz SA, Martín M, Press MF, Chan D, Fernandez-Abad M, Petru E, Rostorfer R, Guarneri V, Huang CS, Barriga S, Wijayawardana S, Brahmachary M, Ebert PJ, et al. Potent Cell-Cycle Inhibition and Upregulation of Immune Response with Abemaciclib and Anastrozole in neoMONARCH, Phase II Neoadjuvant Study in HR+/HER2- Breast Cancer. Clin Cancer Res. 2020; 26:566-80. https://doi.org/10.1158/10780432.CCR-19-1425. [PubMed]

42. Ma CX, Gao F, Luo J, Northfelt DW, Goetz M, Forero A, Hoog J, Naughton M, Ademuyiwa F, Suresh R, Anderson KS, Margenthaler J, Aft R, et al. NeoPalAna: Neoadjuvant Palbociclib, a Cyclin-Dependent Kinase 4/6 Inhibitor, and Anastrozole for Clinical Stage 2 or 3 Estrogen ReceptorPositive Breast Cancer. Clin Cancer Res. 2017; 23:4055-4065. https://doi.org/10.1158/1078-0432.CCR-16-3206. [PubMed]

43. Rosner M, Schipany K, Hengstschläger M. Phosphorylation of nuclear and cytoplasmic pools of ribosomal protein S6 during cell cycle progression. Amino Acids. 2013; 44:12331240. https://doi.org/10.1007/s00726-012-1445-1. [PubMed]

44. Franco J, Balaji U, Freinkman E, Witkiewicz AK, Knudsen ES. Metabolic Reprogramming of Pancreatic Cancer Mediated by CDK4/6 Inhibition Elicits Unique Vulnerabilities. Cell Rep. 2016; 14:979-990. https://doi. org/10.1016/i.celrep.2015.12.094. [PubMed]

45. Zhang J, Xu K, Liu P, Geng Y, Wang B, Gan W, Guo J, Wu F, Chin YR, Berrios C, Lien Evan C, Toker A, DeCaprio James A, et al. Inhibition of Rb Phosphorylation Leads to mTORC2-Mediated Activation of Akt. Mol Cell. 2016; 62:929-942. https://doi.org/10.1016/j.molcel.2016.04.023. [PubMed]

46. Torres-Guzman R, Calsina B, Hermoso A, Baquero C, Alvarez B, Amat J, McNulty AM, Gong X, Boehnke K, Du J, de Dios A, Beckmann RP, Buchanan S, et al. Preclinical characterization of abemaciclib in hormone receptor positive breast cancer. Oncotarget. 2017; 8:69493-69507. https://doi.org/10.18632/oncotarget.17778. [PubMed] 
47. Keane NA, Reidy M, Natoni A, Raab MS, O’Dwyer M. Targeting the Pim kinases in multiple myeloma. Blood Cancer J. 2015; 5:e325. https://doi.org/10.1038/bcj.2015.46. [PubMed]

48. Malinen M, Jääskeläinen T, Pelkonen M, Heikkinen S, Väisänen S, Kosma VM, Nieminen K, Mannermaa A, Palvimo JJ. Proto-oncogene PIM-1 is a novel estrogen receptor target associating with high grade breast tumors. Mol Cell Endocrinol. 2013; 365:270-276. https://doi. org/10.1016/j.mce.2012.10.028. [PubMed]

49. Nawijn MC, Alendar A, Berns A. For better or for worse: the role of Pim oncogenes in tumorigenesis. Nat Rev Cancer. 2011; 11:23-34. https://doi.org/10.1038/nrc2986. [PubMed]

50. Blanco-Aparicio C, Carnero A. Pim kinases in cancer: Diagnostic, prognostic and treatment opportunities. Biochem Pharmacol. 2013; 85:629-643. https://doi. org/10.1016/j.bcp.2012.09.018. [PubMed]

51. Horiuchi D, Camarda R, Zhou AY, Yau C, Momcilovic O, Balakrishnan S, Corella AN, Eyob H, Kessenbrock K, Lawson DA, Marsh LA, Anderton BN, Rohrberg J, et al. PIM1 kinase inhibition as a targeted therapy against triple-negative breast tumors with elevated MYC expression. Nat Med. 2016; 22:1321-1329. https://doi.org/10.1038/nm.4213. [PubMed]

52. Braso-Maristany F, Filosto S, Catchpole S, Marlow R, Quist J, Francesch-Domenech E, Plumb DA, Zakka L, Gazinska P, Liccardi G, Meier P, Gris-Oliver A, Cheang MC, et al. PIM1 kinase regulates cell death, tumor growth and chemotherapy response in triple-negative breast cancer. Nat Med. 2016; 22:1303-1313. https://doi.org/10.1038/nm.4198. [PubMed]

53. Jinesh GG, Mokkapati S, Zhu K, Morales EE. Pim kinase isoforms: devils defending cancer cells from therapeutic and immune attacks. Apoptosis. 2016; 21:1203-1213. https://doi.org/10.1007/s10495-016-1289-3. [PubMed]

54. Chen XP, Losman JA, Cowan S, Donahue E, Fay S, Vuong BQ, Nawijn MC, Capece D, Cohan VL, Rothman P. Pim serine/threonine kinases regulate the stability of Socs-1 protein. Proc Natl Acad Sci U S A. 2002; 99:2175-2180. https://doi.org/10.1073/pnas.042035699. [PubMed]

55. Peltola KJ, Paukku K, Aho TLT, Ruuska M, Silvennoinen O, Koskinen PJ. Pim-1 kinase inhibits STAT5-dependent transcription via its interactions with SOCS1 and SOCS3. Blood. 2004; 103:3744-3750. https://doi.org/10.1182/ blood-2003-09-3126. [PubMed]

56. Goel S, DeCristo MJ, Watt AC. CDK4/6 inhibition triggers anti-tumour immunity. Nature. 2017; 548:471-475. https:// doi.org/10.1038/nature23465. [PubMed]

57. Herrera-Abreu MT, Palafox M, Asghar U, Rivas MA, Cutts RJ, Garcia-Murillas I, Pearson A, Guzman M, Rodriguez O, Grueso J, Bellet M, Cortés J, Elliott R, et al. Early Adaptation and Acquired Resistance to CDK4/6 Inhibition in Estrogen ReceptorPositive Breast Cancer. Cancer Res. 2016; 76:2301-2313. https:// doi.org/10.1158/0008-5472.CAN-15-0728. [PubMed]

58. Rebello RJ, Huglo AV, Furic L. PIM activity in tumours: A key node of therapy resistance. Adv Biol Regul. 2018; 67:163-169. https://doi.org/10.1016/j.jbior.2017.10.010. [PubMed]
59. An N, Xiong Y, LaRue AC, Kraft AS, Cen B. Activation of Pim Kinases Is Sufficient to Promote Resistance to MET Small-Molecule Inhibitors. Cancer Res. 2015; 75:53185328. https://doi.org/10.1158/0008-5472.CAN-15-0544. [PubMed]

60. Henry RE, Barry ER, Castriotta L, Ladd B, Markovets A, Beran G, Ren Y, Zhou F, Adam A, Zinda M, Reimer C, Qing W, Su W, et al. Acquired savolitinib resistance in nonsmall cell lung cancer arises via multiple mechanisms that converge on MET-independent mTOR and MYC activation. Oncotarget. 2016; 7:57651-57670. https://doi.org/10.18632/ oncotarget.10859. [PubMed]

61. Franco J, Witkiewicz AK, Knudsen ES. CDK4/6 inhibitors have potent activity in combination with pathway selective therapeutic agents in models of pancreatic cancer. Oncotarget. 2014; 5:6512-6525. https://doi.org/10.18632/oncotarget.2270. [PubMed]

62. Ku BM, Yi SY, Koh J, Bae YH, Sun JM, Lee SH, Ahn JS, Park K, Ahn MJ. The CDK4/6 inhibitor LY2835219 has potent activity in combination with mTOR inhibitor in head and neck squamous cell carcinoma. Oncotarget. 2016; 7:14803-14813. https://doi.org/10.18632/oncotarget.7543. [PubMed]

63. Ladd B, Mazzola AM, Bihani T, Lai Z, Bradford J, Collins M, Barry E, Goeppert AU, Weir HM, Hearne K, Renshaw JG, Mohseni M, Hurt E, et al. Effective combination therapies in preclinical endocrine resistant breast cancer models harboring ER mutations. Oncotarget. 2016; 7:54120-54136. https://doi.org/10.18632/oncotarget.10852. [PubMed]

64. Olmez I, Brenneman B, Xiao A, Serbulea V, Benamar M, Zhang Y, Manigat L, Abbas T, Lee J, Nakano I, Godlewski J, Bronisz A, Abounader R, et al. Combined CDK4/6 and mTOR inhibition is synergistic against glioblastoma via multiple mechanisms. Clin Cancer Res. 2017; 23:6958-6968. https://doi.org/10.1158/1078-0432.CCR-17-0803. [PubMed]

65. Bonelli MA, Digiacomo G, Fumarola C, Alfieri R, Quaini F, Falco A, Madeddu D, La Monica S, Cretella D, Ravelli A, Ulivi P, Tebaldi M, Calistri D, et al. Combined Inhibition of CDK4/6 and PI3K/AKT/mTOR Pathways Induces a Synergistic Anti-Tumor Effect in Malignant Pleural Mesothelioma Cells. Neoplasia. 2017; 19:637-648. https:// doi.org/10.1016/j.neo.2017.05.003. [PubMed]

66. Zhao L, Wientjes MG, Au JL. Evaluation of combination chemotherapy: integration of nonlinear regression, curve shift, isobologram, and combination index analyses. Clin Cancer Res. 2004; 10:7994-8004. https://doi. org/10.1158/1078-0432.CCR-04-1087. [PubMed]

67. Bliss CI. The toxicity of poisons applied jointly. Ann Appl Biol. 1939; 26:585-615. https://doi. org/10.1111/j.1744-7348.1939.tb06990.x.

68. Barretina J, Caponigro G, Stransky N, Venkatesan K, Margolin AA, Kim S, Wilson CJ, Lehár J, Kryukov GV, Sonkin D, Reddy A, Liu M, Murray L, et al. The Cancer Cell Line Encyclopedia enables predictive modelling of anticancer drug sensitivity. Nature. 2012; 483:603-607. https://doi.org/10.1038/nature11003. [PubMed] 